Full Paper: Mass balance equations in terms of the moment generating function of the distribution of mole concentrations of polymer species for free radical copolymerizations of mono/divinyl monomers could be numerically solved after gel point using open source code ACDC, needed for extremely stiff two-point boundary value problems. For the first time, it became possible to compare the error of earlier wellknown approximated estimation methods for the weight fraction of sol and average molecular weights to this accurate mathematical solution. It turns out that predictions by the pseudo-kinetic method are reasonable only when equal reactivity of double bonds prevails, causing early gelation in the batch reactor. Otherwise the discrepancies between the exact and approximate solutions are quite important.

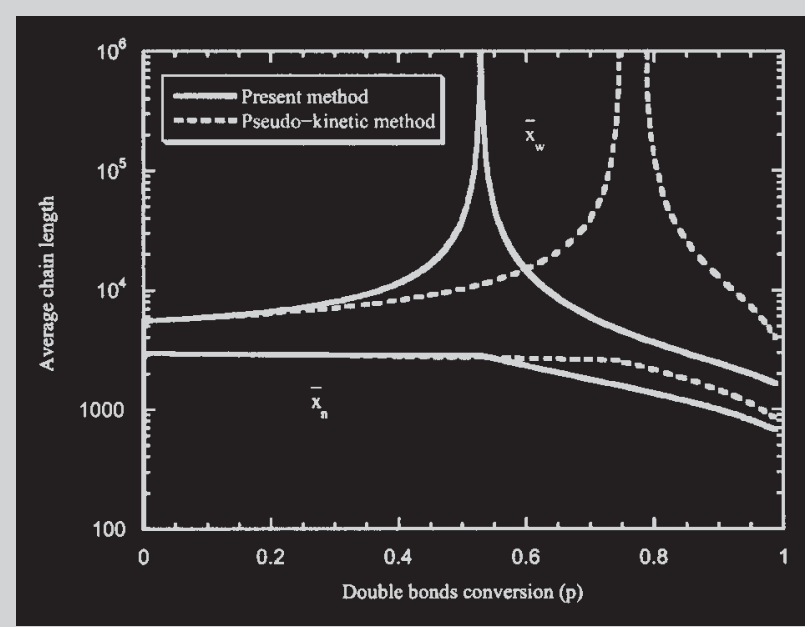

Comparison between predicted number-average and weightaverage degrees of polymerization of sol in batch non-linear free radical polymerizations of model system III.

\title{
Prediction of Sol Fraction and Average Molecular Weights after Gelation for Non-Linear Free Radical Polymerizations Using a Kinetic Approach
}

\author{
Mário Rui P. F. N. Costa, ${ }^{* 1}$ Rolando C. S. Dias ${ }^{2}$ \\ ${ }^{1}$ LSRE, Faculty of Engineering University of Porto, Rua Roberto Frias s/n, 4200-465 Porto Codex, Portugal \\ Fax: 351225081666; E-mail: mrcosta@fe.up.pt \\ ${ }^{2}$ LSRE, Instituto Politécnico de Bragança, Quinta de S. Apolónia, 5300 Bragança, Portugal
}

Keywords: average molecular weights; divinyl monomers; free radical polymerization; gels; kinetics (polym.)

\section{Introduction}

Many key features of kinetic modeling of non-linear free radical polymerizations through the use of the moment generating functions of chain length distributions (CLD) of polymer species have been acknowledged by Kuchanov and Pis'men, ${ }^{[1]}$ namely the need of avoiding the quasi-steady state hypothesis for radical concentrations and the consideration of multiple radical centers near the gelation and beyond.

Lack of suitable numerical methods has precluded the use of this approach for modeling purposes after gelation. The well-known method of moments, which some commercial packages implement, results from similar sets of equations before gel point, but only a limited description of the chemical system is then available.

This kinetic approach for modeling irreversible nonlinear polymerizations has lately been developed by the present authors (Costa and Dias ${ }^{[2]}$ ) with some success as far as non-linear polycondensations and non-radical polyadditions (Costa and Dias ${ }^{[3]}$ ) are concerned. At the same time, some results concerning free radical polymerizations before gelation have been obtained, and some severe numerical difficulties were identified.

It is the purpose of this paper to present a successful way of dealing with those problems and to discuss the value and possible usefulness of this general approach. 


\section{Chemical Modeling}

Polymer molecules are described by the count of their chemical groups regardless of their sequence. Only a very simple kinetic scheme will be considered, in order to present the essential numerical modeling difficulties with a minimum set of equations.

The chemical groups are named $A_{j}$, with $j$ being an integer variable, and in these case studies group $A_{1}$ is a radical site coming from the monovinyl monomer $M_{1}, A_{2}$ is a radical site coming from the divinyl monomer $M_{2}$, and $A_{3}$ is a pending vinyl group. The count of repeating units coming from either monomer, $A_{4}$ and $A_{5}$, is necessary in order to compute molecular weight. Notice that groups $A_{4}$ and $A_{5}$ are only formed, not consumed, by chemical reaction. Remaining active species are the monofunctional initiator $I$ and the primary radicals $R_{0}$ it forms when it decomposes by a first order reaction with rate constant $k_{\mathrm{d}}$ and an efficiency factor $f$ :

$$
I \stackrel{k_{\mathrm{d}}}{\longrightarrow} 2 f R_{0}
$$

Primary radicals initiate kinetic chains by reaction with monomers and pending double bonds:

$$
\begin{aligned}
& R_{0}+M_{1} \stackrel{k_{\mathrm{I}_{1}}}{\longrightarrow} A_{1}+A_{4} \\
& R_{0}+M_{2} \stackrel{k_{\mathrm{I}_{2}}}{\longrightarrow} A_{2}+A_{3}+A_{5} \\
& R_{0}+A_{3} \stackrel{k_{\mathrm{I}_{3}}}{\longrightarrow} A_{2}
\end{aligned}
$$

Considering only the effect of the terminal unit of the radicals in the reactivity, there are six different propagation reactions:

$$
\begin{aligned}
& A_{1}+M_{1} \stackrel{k_{\mathrm{p} 11}}{\longrightarrow} A_{1}+A_{4} \\
& A_{1}+M_{2} \stackrel{k_{\mathrm{p} 12}}{\longrightarrow} A_{2}+A_{3}+A_{5} \\
& A_{1}+A_{3} \stackrel{k_{\mathrm{p}_{13}}}{\longrightarrow} A_{2} \\
& A_{2}+M_{1} \stackrel{k_{\mathrm{p} 21}}{\longrightarrow} A_{1}+A_{4} \\
& A_{2}+M_{2} \stackrel{k_{\mathrm{p} 22}}{\longrightarrow} A_{2}+A_{3}+A_{5} \\
& A_{2}+A_{3} \stackrel{k_{\mathrm{p} 23}}{\longrightarrow} A_{2}
\end{aligned}
$$

Accordingly, there are six different termination reactions, half by combination and half by dismutation:
$A_{1}+A_{1} \stackrel{k_{\mathrm{tc}_{11}}}{\longrightarrow}$ Head - Head unit

$A_{1}+A_{1} \stackrel{k_{\mathrm{td}_{11}}}{\longrightarrow}$ Saturated endgroup + Unsaturated endgroup

$A_{1}+A_{2} \stackrel{k_{\mathrm{t}_{12}}}{\longrightarrow}$ Head - Head unit

$A_{1}+A_{2} \stackrel{k_{\mathrm{td}_{12}}}{\longrightarrow}$ Saturated endgroup + Unsaturated endgroup

$A_{2}+A_{2} \stackrel{k_{\mathrm{tc}_{22}}}{\longrightarrow}$ Head - Head unit

$A_{2}+A_{2} \stackrel{k_{\mathrm{t}_{22}}}{\longrightarrow}$ Saturated endgroup + Unsaturated endgroup

No less than three different conventions for relating rates of group formation with the values of rate constants of termination can be found in the literature. In the convention we follow, the rate of consumption of $A_{1}$ radical groups terminating by combination with themselves is $2 k_{\mathrm{tc}_{11}} A_{1}^{2}$ and rate of formation of head-to-head units is $k_{\mathrm{tc}_{11}} A_{1}^{2}$. In the same way, rate of consumption of $A_{1}$ radical groups by dismutation is $2 k_{\mathrm{td}_{11}} A_{1}^{2}$.

The set of polymer species with $a_{1}$ groups $A_{1}$ (radical sites from monomer $\left.M_{1}\right), a_{2}$ radicals $A_{2}$ and $a_{3}$ pending double bonds $A_{3}, a_{4}$ units of monomer " 1 " and $a_{5}$ units of monomer " 2 " ( $a_{4}$ and $a_{5}$ are the degrees of polymerization) is supposed to have an overall mole concentration $P\left(a_{1}, a_{2}\right.$, $\left.a_{3}, a_{4}, a_{5}\right)$. We will often use its moment generating function $G\left(s_{1}, s_{2}, s_{3}, s_{4}, s_{5}\right)$ below defined:

$$
\begin{aligned}
& G\left(s_{1}, s_{2}, s_{3}, s_{4}, s_{5}\right)=\sum_{a_{1}=0}^{\infty} \sum_{a_{2}=0}^{\infty} \sum_{a_{3}=0}^{\infty} \sum_{a_{4}=0}^{\infty} \sum_{a_{5}=0}^{\infty} s_{1}^{a_{1}} s_{2}^{a_{2}} s_{3}^{a_{3}} s_{4}^{a_{4}} s_{5}^{a_{5}} \\
& \quad \times P\left(a_{1}, a_{2}, a_{3}, a_{4}, a_{5}\right)
\end{aligned}
$$

Laplace parameters $s_{j}$, with $j=1-5$, are complex numbers. As $G(1,1,1,1,1)$ is some finite value equal to the overall mole concentration of polymer, the comparison test guarantees convergence of the series in Equation (17) in the five-dimensional sphere in complex space $|\mathbf{s}| \leq 1$, moment generating function $G$ being thus an analytical function of those parameters for $|\mathbf{s}|<1$.

Partial moments with respect to the counts of groups are obtained through differentiation of Equation (17) with respect to the $\log s_{j}$ followed by setting all values of Laplace parameters equal to 1 . The notation $G_{i j . . . k}=$ $\frac{\partial \partial \ldots \partial G}{\partial \log s_{i} \partial \log s_{j} \ldots \partial \log s_{k}}$ will often be used for those derivatives (notice that the indices $i, j, \ldots k$ may be repeated any number 
of times), leading to:

$\sum_{a_{1}=0}^{\infty} \sum_{a_{2}=0}^{\infty} \cdots \sum_{a_{5}=0}^{\infty} a_{i} a_{j} \cdots a_{k} P\left(a_{1}, \ldots, a_{5}\right)=G_{i j \ldots k}(1,1,1,1,1)$

It might also be possible to use fast Fourier transform to numerically invert $G^{[2]}$ One could obtain the full multidimensional or any sub-set of marginal distributions given a sufficiently large number (a few thousands or tens of thousands per variable) of equally spaced values of $G$ in a sphere in complex five-dimensional space centered in the origin of radius slightly below 1 . Too high computing time needed to obtain those required values of $G$ with current implementation of numerical methods, as will be discussed with more detail towards the end of this paper, precluded the use of this approach in the current work.

Using techniques we have presented before, ${ }^{[2]}$ for a batch reactor with negligible volume change and no chain-length dependence of rate constants, the moment generating function of mass balance of polymer species verifies the partial differential equation shown below: $\frac{\mathrm{d} M_{2}}{\mathrm{~d} t}=-k_{\mathrm{I}_{2}} R_{0} M_{2}-k_{\mathrm{p}_{12}} A_{1} M_{2}-k_{\mathrm{p}_{22}} A_{2} M_{2}$

$\frac{\mathrm{d} R_{0}}{\mathrm{~d} t}=2 f k_{\mathrm{d}} I-\left(k_{\mathrm{I}_{1}} M_{1}+k_{\mathrm{I}_{2}} M_{2}+k_{\mathrm{I}_{3}} A_{3}\right) R_{0}$

$\frac{\mathrm{d} I}{\mathrm{~d} t}=-k_{\mathrm{d}} I$

with initial conditions (assuming there are only monomers and initiator at the beginning):

$$
\begin{aligned}
& G_{\mid t=0}=0 \\
& A_{j_{\mid t=0}}=0 \quad j=1 \ldots 3 \\
& M_{\left.\right|_{t=0}}=M_{1_{0}} \\
& M_{2_{\mid t=0}}=M_{2_{0}} \\
& R_{\left.0\right|_{t=0}}=0
\end{aligned}
$$

$$
\begin{aligned}
\frac{\partial G}{\partial t}= & k_{\mathrm{p}_{13}}\left(\frac{s_{2}}{s_{1} s_{3}} \frac{\partial G}{\partial \log s_{1}} \frac{\partial G}{\partial \log s_{3}}-\frac{\partial G}{\partial \log s_{1}} A_{3}-\frac{\partial G}{\partial \log s_{3}} A_{1}\right)+k_{\mathrm{p}_{23}}\left(\frac{1}{s_{3}} \frac{\partial G}{\partial \log s_{2}} \frac{\partial G}{\partial \log s_{3}}-\frac{\partial G}{\partial \log s_{2}} A_{3}-\frac{\partial G}{\partial \log s_{3}} A_{2}\right) \\
& +k_{\mathrm{tc}_{11}}\left[\frac{1}{s_{1}^{2}}\left(\frac{\partial G}{\partial \log s_{1}}\right)^{2}-2 \frac{\partial G}{\partial \log s_{1}} A_{1}\right]+k_{\mathrm{tc} 22}\left[\frac{1}{s_{2}^{2}}\left(\frac{\partial G}{\partial \log s_{2}}\right)^{2}-2 \frac{\partial G}{\partial \log s_{2}} A_{2}\right] \\
& +k_{\mathrm{tc}_{12}}\left(\frac{1}{s_{1} s_{2}} \frac{\partial G}{\partial \log s_{1}} \frac{\partial G}{\partial \log s_{2}}-\frac{\partial G}{\partial \log s_{1}} A_{2}-\frac{\partial G}{\partial \log s_{2}} A_{1}\right)+k_{\mathrm{p}_{11}} \frac{\partial G}{\partial \log s_{1}} M_{1}\left(s_{4}-1\right)+k_{\mathrm{p}_{12}} \frac{\partial G}{\partial \log s_{1}} M_{2}\left(\frac{s_{2} s_{3} s_{5}}{s_{1}}-1\right) \\
& +k_{\mathrm{p}_{21}} \frac{\partial G}{\partial \log s_{2}} M_{1}\left(\frac{s_{1} s_{4}}{s_{2}}-1\right)+k_{\mathrm{p}_{22}} \frac{\partial G}{\partial \log s_{2}} M_{2}\left(s_{3} s_{5}-1\right)+2 k_{\mathrm{td}} \frac{\partial G}{\partial \log s_{1}} A_{1}\left(\frac{1}{s_{1}}-1\right) \\
& +k_{\mathrm{td}_{12}}\left[\frac{\partial G}{\partial \log s_{1}} A_{2}\left(\frac{1}{s_{1}}-1\right)+\frac{\partial G}{\partial \log s_{2}} A_{1}\left(\frac{1}{s_{2}}-1\right)\right]+2 k_{\mathrm{td}} \frac{\partial G}{\partial \log s_{2}} A_{2}\left(\frac{1}{s_{2}}-1\right) \\
& +k_{\mathrm{I}_{3}} \frac{\partial G}{\partial \log s_{3}} R_{0}\left(\frac{s_{2}}{s_{3}}-1\right)+k_{\mathrm{I}_{1}} M_{1} R_{0} s_{1} s_{4}+k_{\mathrm{I}_{2}} M_{2} R_{0} s_{2} s_{3} s_{5}
\end{aligned}
$$

$$
\begin{aligned}
\frac{\mathrm{d} A_{1}}{\mathrm{~d} t}= & k_{\mathrm{I}_{1}} R_{0} M_{1}-k_{\mathrm{p}_{12}} A_{1} M_{2}+k_{\mathrm{p}_{21}} A_{2} M_{1}-k_{\mathrm{p}_{13}} A_{1} A_{3} \\
& -2 k_{\mathrm{tc}_{11}} A_{1}^{2}-k_{\mathrm{tc}_{12}} A_{1} A_{2}-2 \mathrm{k}_{\mathrm{td}_{11}} A_{1}^{2}-k_{\mathrm{td}_{12}} A_{1} A_{2} \\
\frac{\mathrm{d} A_{2}}{\mathrm{~d} t}= & k_{\mathrm{I}_{2}} R_{0} M_{2}+k_{\mathrm{I}_{3}} R_{0} A_{3}+k_{\mathrm{p}_{12}} A_{1} M_{2}-k_{\mathrm{p}_{21}} A_{2} M_{1}+k_{\mathrm{p}_{13}} A_{1} A_{3} \\
& -2 k_{\mathrm{tc}_{22}} A_{2}^{2}-k_{\mathrm{tc}_{12}} A_{1} A_{2}-2 \mathrm{k}_{\mathrm{td}_{22}} A_{2}^{2}-k_{\mathrm{td}_{12}} A_{1} A_{2}
\end{aligned}
$$

$$
\begin{aligned}
\frac{\mathrm{d} A_{3}}{\mathrm{~d} t}= & k_{\mathrm{I}_{2}} R_{0} M_{2}-k_{\mathrm{I}_{3}} R_{0} A_{3}+k_{\mathrm{p}_{12}} A_{1} M_{2}+k_{\mathrm{p}_{22}} A_{2} M_{2} \\
& -k_{\mathrm{p}_{13}} A_{1} A_{3}-k_{\mathrm{p}_{23}} A_{2} A_{3} \\
\frac{\mathrm{d} M_{1}}{\mathrm{~d} t}= & -k_{\mathrm{I}_{1}} R_{0} M_{1}-k_{\mathrm{p}_{11}} A_{1} M_{1}-k_{\mathrm{p}_{21}} A_{2} M_{1}
\end{aligned}
$$

$$
I_{\mid t=0}=I_{0}
$$

The solution of Equation (19) together with ordinary differential Equation (20)-(26) and their initial conditions, Equation (27)-(32), is obtained by the method of characteristics (Courant and Hilbert ${ }^{[4]}$ ) as explained in more detail elsewhere. ${ }^{[2]}$ The characteristics are the solution of the system below:

$$
\begin{aligned}
\frac{d G_{1}}{d t}= & k_{\mathrm{I}_{1}} R_{0} M_{1} s_{1}-k_{\mathrm{p}_{12}} \frac{s_{2} s_{3}}{s_{1}} G_{1} M_{2}+k_{\mathrm{p}_{21}} \frac{s_{1}}{s_{2}} G_{2} M_{1} \\
& -k_{\mathrm{p}_{13}} \frac{s_{2}}{s_{1} s_{3}} G_{1} G_{3}-2 k_{\mathrm{tc}_{11}} \frac{G_{1}^{2}}{s_{1}^{2}}-k_{\mathrm{tc}_{12}} \frac{G_{1} G_{2}}{s_{1} s_{2}} \\
& -2 k_{\mathrm{td}_{11}} A_{1} \frac{G_{1}}{s_{1}}-k_{\mathrm{td}_{12}} A_{2} \frac{G_{1}}{s_{1}}
\end{aligned}
$$




$$
\begin{aligned}
\frac{\mathrm{d} G_{2}}{\mathrm{~d} t}= & k_{\mathrm{I}_{2}} R_{0} M_{2} s_{2} s_{3}+k_{\mathrm{I}_{3}} \frac{s_{2}}{s_{3}} R_{0} G_{3}+k_{\mathrm{p}_{12}} \frac{s_{2} s_{3}}{s_{1}} G_{1} M_{2} \\
& -k_{\mathrm{p}_{21}} \frac{s_{1}}{s_{2}} G_{2} M_{1}+k_{\mathrm{p}_{13}} \frac{s_{2}}{s_{1} s_{3}} G_{1} G_{3}-2 k_{\mathrm{tc}_{22}} \frac{G_{2}^{2}}{s_{2}^{2}} \\
& -k_{\mathrm{tc}_{12}} \frac{G_{1} G_{2}}{s_{1} s_{2}}-2 k_{\mathrm{td}_{22}} A_{2} \frac{G_{2}}{s_{2}}-k_{\mathrm{td}_{12}} A_{1} \frac{G_{2}}{s_{2}}
\end{aligned}
$$

$$
\begin{aligned}
\frac{\mathrm{d} G_{3}}{\mathrm{~d} t}= & k_{\mathrm{I}_{2}} R_{0} M_{2} s_{2} s_{3}-k_{\mathrm{I}_{3}} \frac{s_{2}}{s_{3}} R_{0} G_{3}+k_{\mathrm{p}_{12}} \frac{s_{2} s_{3}}{s_{1}} G_{1} M_{2} \\
& +k_{\mathrm{p}_{22}} s_{3} G_{2} M_{2}-k_{\mathrm{p}_{13}} \frac{s_{2}}{s_{1} s_{3}} G_{1} G_{3}-k_{\mathrm{p}_{23}} \frac{G_{2} G_{3}}{s_{3}}
\end{aligned}
$$

$$
\begin{aligned}
\frac{\mathrm{d} s_{1}}{\mathrm{~d} t}= & k_{\mathrm{p}_{13}}\left(s_{1} A_{3}-\frac{s_{2}}{s_{3}} G_{3}\right)+k_{\mathrm{p}_{12}} M_{2}\left(s_{1}-s_{2} s_{3}\right) \\
& +2 k_{\mathrm{tc}_{11}}\left(s_{1} A_{1}-\frac{G_{1}}{s_{1}}\right)+k_{\mathrm{tc}_{12}}\left(s_{1} A_{2}-\frac{G_{2}}{s_{2}}\right) \\
& +2 k_{\mathrm{td}_{11}} A_{1}\left(s_{1}-1\right)+k_{\mathrm{td}_{12}} A_{2}\left(s_{1}-1\right)
\end{aligned}
$$

$$
\begin{aligned}
\frac{\mathrm{d} s_{2}}{\mathrm{~d} t}= & k_{\mathrm{p}_{23}}\left(s_{2} A_{3}-\frac{s_{2}}{s_{3}} G_{3}\right)+k_{\mathrm{p}_{21}} M_{1}\left(s_{2}-s_{1}\right) \\
& +k_{\mathrm{p}_{22}} M_{2}\left(s_{2}-s_{2} s_{3}\right)+2 k_{\mathrm{tc}_{22}}\left(s_{2} A_{2}-\frac{G_{2}}{s_{2}}\right) \\
& +k_{\mathrm{tc}_{12}}\left(s_{2} A_{1}-\frac{G_{1}}{s_{1}}\right)+2 k_{\mathrm{td}_{22}} A_{2}\left(s_{2}-1\right) \\
& +k_{\mathrm{td}_{12}} A_{1}\left(s_{2}-1\right)
\end{aligned}
$$

$$
\begin{aligned}
\frac{\mathrm{d} s_{3}}{\mathrm{~d} t}= & k_{\mathrm{I}_{3}} R_{0}\left(s_{3}-s_{2}\right)+k_{\mathrm{p}_{13}}\left(s_{3} A_{1}-\frac{s_{2}}{s_{1}} G_{1}\right) \\
& +k_{\mathrm{p}_{23}}\left(s_{3} A_{2}-G_{2}\right)
\end{aligned}
$$

$$
\begin{aligned}
\frac{\mathrm{d} G}{\mathrm{~d} t}= & k_{\mathrm{I}_{1}} R_{0} M_{1} s_{1}+k_{\mathrm{I}_{2}} R_{0} M_{2} s_{2} s_{3}-k_{\mathrm{p}_{13}} \frac{s_{2}}{s_{1} s_{3}} G_{1} G_{3} \\
& -k_{\mathrm{p}_{23}} \frac{G_{2} G_{3}}{s_{3}}-k_{\mathrm{tc}_{11}} \frac{G_{1}^{2}}{s_{1}^{2}}-k_{\mathrm{tc}_{12}} \frac{G_{1} G_{2}}{s_{1} s_{2}}-k_{\mathrm{tc}_{22}} \frac{G_{2}^{2}}{s_{2}^{2}}
\end{aligned}
$$

With further initial conditions:

$$
\begin{gathered}
G_{\left.j\right|_{t=0}}=0 \quad j=1 \ldots 3 \\
s_{\left.j\right|_{t=0}}=s_{j_{0}} \quad j=1 \ldots 3
\end{gathered}
$$

Since the goal is computing the value of $G$ and, very often, some of its derivatives with respect to the Laplace parameters at some prescribed time $t_{f}$ and vector of the $s_{j}$, $\mathbf{s}_{\mathbf{f}}=\left[s_{j_{f}}\right]$, one has to solve a two point boundary value problem with the conditions at the right point $t=t_{f}$ :

$$
s_{\left.j\right|_{t=t_{f}}}=s_{j_{f}} \quad j=1 \ldots 3
$$

If the method for computing CLD by fast Fourier transform as described in ref. ${ }^{[2]}$ is to be used, the vector $\mathbf{S}_{\mathbf{f}}$ shall lie over a spherical surface in complex space centered in the origin and with radius $1\left(\left|\mathbf{s}_{\mathbf{f}}\right|=1\right)$ or slightly less than 1. But more often, the goal is only to compute the moments of CLD and therefore all $s_{j_{f}}=1$.

Before gelation, the physically meaningful solution when all $s_{j_{f}}=1$ (or $\left.\mathbf{s}_{\mathbf{f}}=\mathbf{1}\right)$ is $s_{j_{0}}=1$ for all $j\left(\right.$ or $\mathbf{s}_{\mathbf{0}}=\mathbf{1}$ ); all $s_{j}$ are constant along the characteristic lines. This is what we call thereafter the trivial solution branch.

Gel point is a bifurcation point where a second solution branch with at least some $s_{j_{0}}<1$ intersects the trivial solution for $t=t_{g}$, the gelation time.

For $t_{f}>t_{g}$ the physically meaningful solution is the nontrivial solution branch.

After gelation, this minimum problem can not be avoided and its solution is not easy at all. We will discuss next a practical way of solving it, which has worked for this relatively simple chemical system.

\section{Numerical Solution of the Minimum System for Characteristics}

Laplace parameters associated to non-reactive groups, such as $s_{4}$ and $s_{5}$ in this example, are constant along the characteristics. In this study, chain length distributions will not be computed, only their moments, and so they will be set to 1 for the rest of this presentation.

This particular class of boundary value problems is of the "time-like" variety in Deuflhard's classification. ${ }^{[5]} \mathrm{A}$ shooting method using integration for increasing time values is the natural way of finding a numerical solution, as we have used previously. ${ }^{[2,3]}$

Because of the high relative values of termination rate constants relatively to propagation, it can be guessed that the system becomes extremely "stiff", ${ }^{[6]}$ and this can be verified in a number of ways.

A widely used measure of numerical stiffness ${ }^{[7]}$ is found by multiplying the end value of time $t_{f}$ by the lowest real part of the eigenvalues of the Jacobian of the system right hand sides. After obtaining an accurate numerical solution of Equation (20)-(26) and (33)-(39), as will be described later on, this criterion can be evaluated. It increases rapidly in modulus with time (being already $-10^{6}$ at double bonds conversion of 0.01 ), and it reaches values of about $-2 \times 10^{7}$ for the numerical case studies next presented.

Well known code for "stiff" systems of ordinary differential equations RADAU5 ${ }^{[8]}$ can successfully solve the system consisting only of the end-group mass balances, Equation (20)-(26). However, on trying to use that method to numerically solve the full system with additional Equation (33)-(38), it turns out that numerical values "blow out" even with the exact solution (trivial solution branch) for the initial values of $s_{j_{0}}$ (Figure 1)!

Therefore, parallel multiple shooting ${ }^{[9]}$ is needed to overcome this difficulty. 


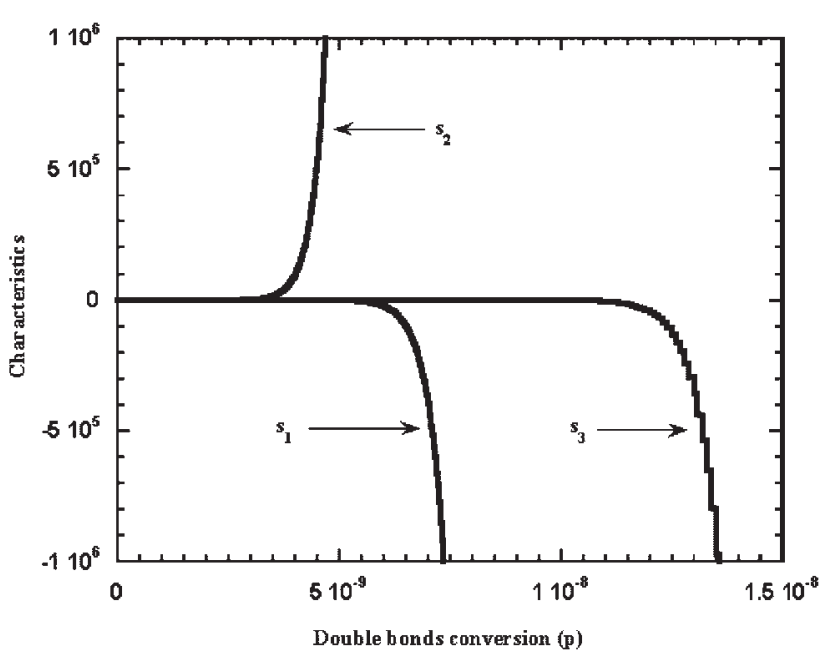

Figure 1. Example of integration of characteristics showing the "blow up" of numerical solution.

The extreme numerical sensitivity of these problems made all currently available codes fail, except the integrator in Cash et al.'s automatic continuation code ACDC, ${ }^{[10-12]}$ available at http://www.netlib.org/ode/acdc.f. Although its continuation procedure (in its present form) usually leads to the trivial solution branch instead of the physically meaningful branch, its implicit Runge-Kutta integrator based on a Lobatto quadrature does succeed in finding the required solutions.

Three sets of numerical values for parameters used in simulations are presented in Table 1. A few common values for the three systems are representative ${ }^{[13]}$ of real freeradical polymerizations. Some ratios of constants, including the well-known copolymerization ratios $r_{1}$ and $r_{2}$ for the classical terminal unit effect, were chosen in order to explore numerical sensitivities.

Table 1. Values of rate parameters used in simulations.

\begin{tabular}{|c|c|c|c|}
\hline Parameter & $\begin{array}{c}\text { System } \\
\text { I }\end{array}$ & $\begin{array}{c}\text { System } \\
\text { II }\end{array}$ & $\begin{array}{c}\text { System } \\
\text { III }\end{array}$ \\
\hline$C_{\mathrm{I}_{1}}=k_{\mathrm{I}_{1}} / k_{\mathrm{p}_{11}}$ & 1 & 1 & 1 \\
\hline$C_{\mathrm{I}_{2}}=k_{\mathrm{I}_{2}} / k_{\mathrm{p}_{11}}$ & 2 & 5 & 20 \\
\hline$C_{\mathrm{I}_{3}}=k_{\mathrm{I}_{3}} / k_{\mathrm{p}_{11}}$ & 1 & 0.5 & 10 \\
\hline$r_{1}=k_{\mathrm{p}_{11}} / k_{\mathrm{p}_{12}}$ & 0.5 & 0.2 & 2 \\
\hline$r_{2}=k_{\mathrm{p}_{22}} / k_{\mathrm{p}_{21}}$ & 2 & 0.5 & 500 \\
\hline$C_{\mathrm{p}_{1}}=k_{\mathrm{p}_{21}} / k_{\mathrm{p}_{11}}$ & 1 & 1 & 0.2 \\
\hline$C_{\mathrm{p}_{2}}=k_{\mathrm{p}_{13}} / k_{\mathrm{p}_{11}}$ & 1 & 0.15 & 0.1 \\
\hline$C_{\mathrm{p}_{3}}^{\mathrm{P}_{2}}=k_{\mathrm{p}_{23}} / k_{\mathrm{p}_{21}}$ & 1 & 0.05 & 250 \\
\hline$f$ & 0.5 & 0.5 & 0.5 \\
\hline$k_{\mathrm{d}} / \mathrm{s}^{-1}$ & $10^{-6}$ & $10^{-6}$ & $10^{-6}$ \\
\hline$k_{\mathrm{p}_{11}} /\left(\mathrm{dm}^{3} \cdot \mathrm{mol}^{-1} \cdot \mathrm{s}^{-1}\right)$ & 100 & 100 & 100 \\
\hline$k_{\mathrm{t}_{11}}=k_{\mathrm{tc}_{11}}+k_{\mathrm{td}_{11}} /\left(\mathrm{dm}^{3} \cdot \mathrm{mol}^{-1} \cdot \mathrm{s}^{-1}\right)$ & $10^{6}$ & $10^{6}$ & $10^{6}$ \\
\hline$k_{\mathrm{t}_{12}}=k_{\mathrm{tc}_{12}}+k_{\mathrm{td}_{12}} /\left(\mathrm{dm}^{3} \cdot \mathrm{mol}^{-1} \cdot \mathrm{s}^{-1}\right)$ & $10^{6}$ & $10^{6}$ & $10^{6}$ \\
\hline$k_{\mathrm{t}_{22}}=k_{\mathrm{tc}_{22}}+k_{\mathrm{td}_{22}} /\left(\mathrm{dm}^{3} \cdot \mathrm{mol}^{-1} \cdot \mathrm{s}^{-1}\right)$ & $10^{6}$ & $10^{6}$ & $10^{6}$ \\
\hline$\delta_{\mathrm{c}_{11}}=k_{\mathrm{tc}_{11}} / k_{\mathrm{t}_{11}}$ & 0.5 & 0.5 & 0.5 \\
\hline$\delta_{\mathrm{c}_{12}}=k_{\mathrm{tc}_{12}} / k_{\mathrm{t}_{12}}$ & 0.5 & 0.5 & 0.5 \\
\hline$\delta_{\mathrm{c}_{22}}=k_{\mathrm{tc}_{22}} / k_{\mathrm{t}_{22}}$ & 0.5 & 0.5 & 0.5 \\
\hline
\end{tabular}

System I is the "ideal" base system $\left(r_{1} r_{2}=1\right)$, whereas in system II cross-polymerization is favored $\left(r_{1} r_{2}=0.1\right)$ and nearly forbidden in system III $\left(r_{1} r_{2}=1000\right)$.

In all simulations, the initial composition is the same:

i) initiator concentration is $1 / 1000$ of the double bonds;

ii) divinyl monomer mole fraction is 0.001 with respect to the total monomer content.

Especially important is the effect of the relative reactivity of pendent double bond (determined by $C_{\mathrm{p}_{2}}$ and $C_{\mathrm{p}_{3}}$ parameters). These parameters were varied in order to obtain a lower or higher value of conversion of double bonds at the gel point.

The initial total monomer concentration was fixed to $1 \mathrm{~mol} \cdot \mathrm{dm}^{-3}$.

Code ACDC requires subroutines for defining right hand sides of the system of 13 differential equations, Equation (20)-(26) and (33)-(38), boundary conditions at the two end-points $t=0$ and $t=t_{f}$, Equation (28)-(32), (40), and (42), as well as their Jacobians. Jacobian of right-hand sides of those differential equations has 90 non-zero components.

The first step of the simulations is the prediction of gelation time, since it makes no sense to find again the known trivial solution branch. Both natural ways of carrying out this calculation were used:

1) Integration of the equations for the 2 nd order moments (along the trivial solution branch) until divergence to infinity;

2) Evaluation of the determinant of the Jacobian of the $s_{j}$ relatively to the $s_{j_{0}}$ as a function of time $t$ until it becomes zero, for $t=t_{g}$.

After finding gelation time, a vector of final times will be chosen for the presentation of results, although for easier comparison of chemical systems, overall double bonds conversion will be used instead of the corresponding reaction time.

ACDC computes iteratively the characteristics in a mesh of intermediate time values between $t=0$ and $t=t_{f}$. It adapts the mesh along this process, but a good initial definition of number and placement of the grid is important, and a good approximation of the true solution can be of a great help.

For the first value of final time $t_{f}$, there is no information concerning the values of the $s_{j_{0}}$, except that they should be slightly a bit less than 1 if $t_{f}$ is not far from $t_{g}$, although this does not help much. Fortunately, ACDC finds the wanted solution without many retrials, if one respects the following rules:

The integration interval from $t=0$ to $t=t_{f}$ must be divided into a fairly large number of grid points, with 3 sections, because there are two boundary layers for the solution in each side of the interval, as shown in Figure 2; good choices were 1000,20000 and 1000, with break points $t=t_{s}$ and $t_{f}-t_{s}$, with $t_{s}=100 \mathrm{~s}$ for these numerical examples. $t_{s}$ is of the order of the time needed to reach a slowly changing radical concentration. 


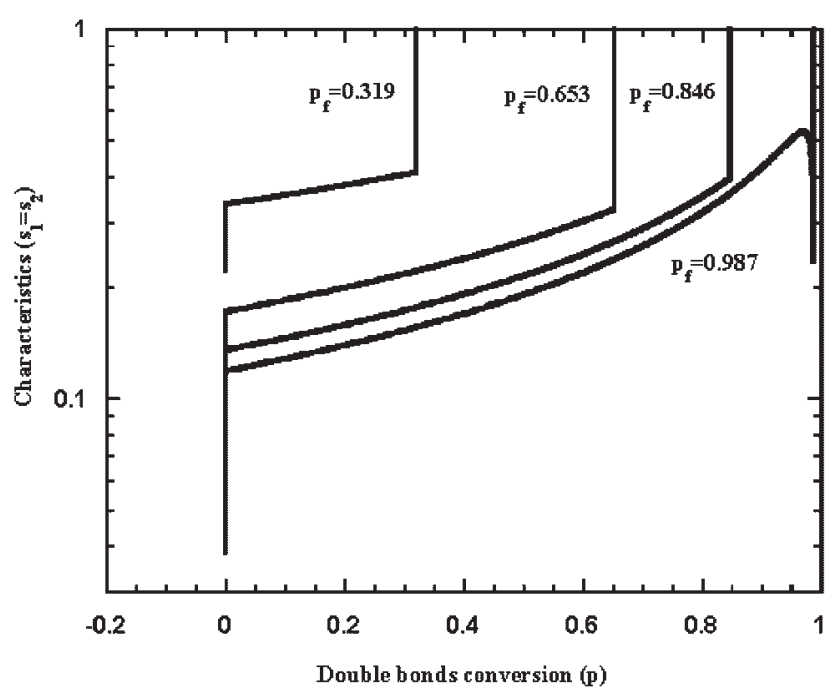

Figure 2. Values of $s_{1}=s_{2}$ along the characteristics for system I and different values of final time (expressed as conversion of double bonds).

In these grid points, as the exact values of the concentrations of active groups can be independently computed, they must be fed to the routine as starting points in order to accelerate convergence.

Initial estimations for $G_{1}, G_{2}$, and $G_{3}$ on the grid points were chosen as some fraction (0.001 gave good results) of the corresponding concentrations $A_{1}, A_{2}$, and $A_{3}$.

Initial estimations for $s_{1}, s_{2}$, and $s_{3}$ on the grid points should follow a pattern similar to the solution in Figure 2: in the left boundary layer, a small value like $10^{-5}$, in the middle region 0.001 or so, and 1 in the right boundary layer.

It took a fairly large number of failed attempts of convergence until these basic rules were found and could thus provide decisive help in finding the solutions with a good reliability now.

In Figure 3, a typical profile of characteristics associated to the different active groups for system III can be observed. Only, for this system, values of $s_{1}$ and $s_{2}$ are very different.

Contrarily to common sense, there is no visible advantage of using a previously found numerical solution as a starting point of the one for the next final value of time. Figure 4 shows why: the changes of profiles are so sudden that one should use extremely close values of successive final times in order for that strategy to succeed. Each prediction of a certain value of final time is thus a nearly independent problem.

The computations were carried out using a PIV $1.5 \mathrm{GHz}$ computer with $512 \mathrm{Mb}$ memory, running Red Hat Linux 7.1 (kernel version 2.4.7-10). Fortran sources were compiled using GNU compiler g77 based on gcc 2.96. In the worst cases, CPU time spent for integrating the characteristics in order to attain each prescribed final time was $270 \mathrm{~s}$ after gelation. Predicting average molecular weights vs. time

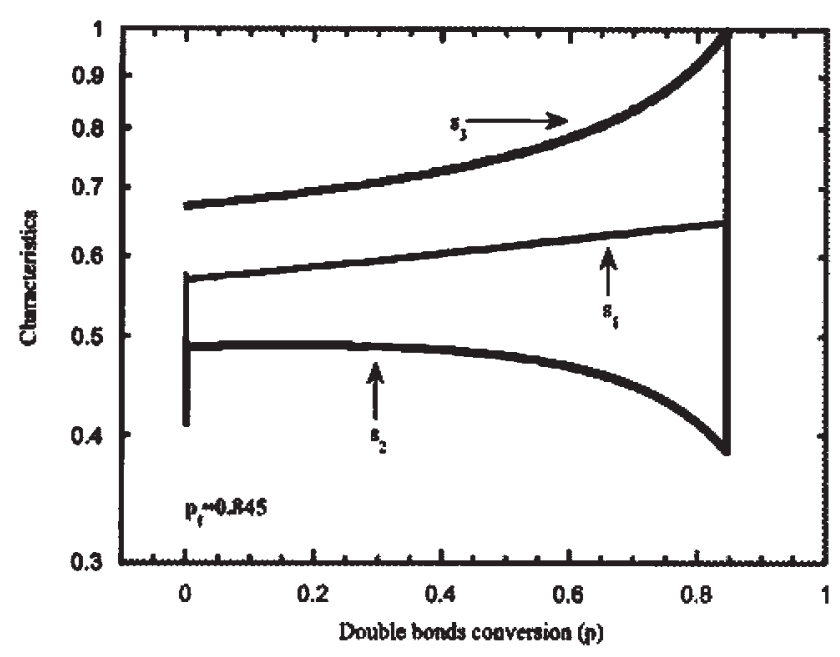

Figure 3. Profiles of $s_{1}, s_{2}$, and $s_{3}$ along the characteristics for system III at conversion of double bonds 0.845 .

before gelation is a much easier problem, only $17 \mathrm{~s}$ are needed for performing the whole computation.

Memory use reached $60 \%$ of the available; this is a likely bottleneck with more complex problems.

\section{Prediction of Sol Fraction and Average Molecular Weights}

Computing higher derivatives of Equation (19) with respect to $\log s_{j}$ yields a system of first-order partial differential equations sharing the same characteristics. In order to predict weight fraction of sol, the moments with respect to the numbers of units $A_{4}$ and $A_{5}$ must be known. They result

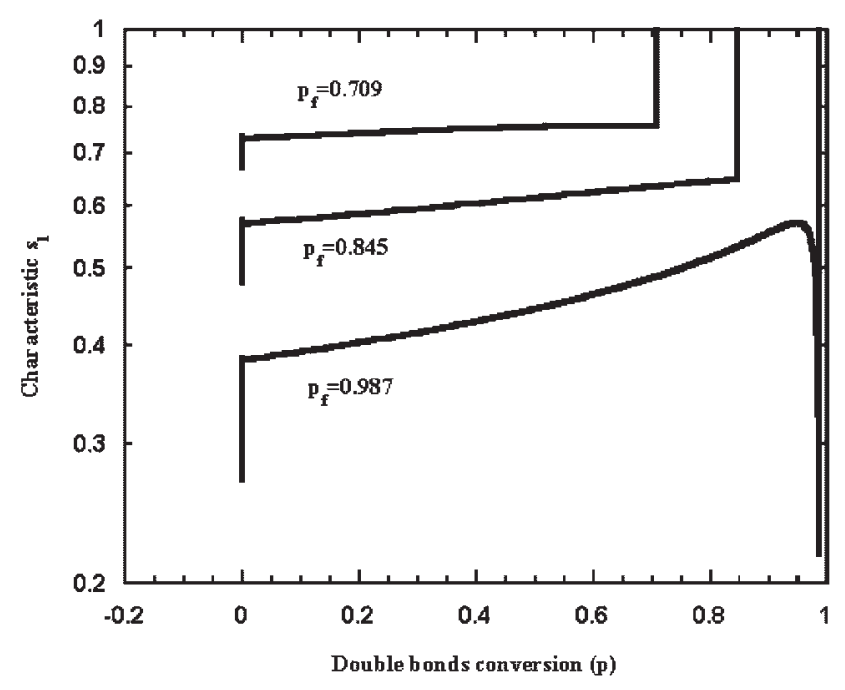

Figure 4. Profiles of $s_{1}$ along the characteristics for system III and different values of final conversion of double bonds. 
from integrating the two differential equations below along the characteristics:

$\frac{\mathrm{d} G_{4}}{\mathrm{~d} t}=k_{\mathrm{I}_{1}} R_{0} M_{1} s_{1}+k_{\mathrm{p}_{11}} G_{1} M_{1}+k_{\mathrm{p}_{21}} \frac{s_{1}}{s_{2}} G_{2} M_{1}$

$\frac{\mathrm{d} G_{5}}{\mathrm{~d} t}=k_{\mathrm{I}_{2}} s_{2} s_{3} R_{0} M_{2}+k_{\mathrm{p}_{12}} \frac{s_{2} s_{3}}{s_{1}} G_{1} M_{2}+k_{\mathrm{p}_{22}} s_{3} G_{2} M_{2}$

$G_{\left.j\right|_{t=0}}=0 \quad j=4,5$

Weight fraction of sol $w_{\mathrm{s}}$ is thus:

$w_{\mathrm{s}}=\frac{M_{M_{1}} G_{4}(\mathbf{1})+M_{M_{2}} G_{5}(\mathbf{1})}{M_{M_{1}}\left(M_{1_{0}}-M_{1}\right)+M_{M_{2}}\left(M_{2_{0}}-M_{2}\right)}$

Equation (43) and (44) must be integrated after successful convergence of the boundary value problem, which determines the characteristics. The solution vector of $s_{1}, s_{2}$, and $s_{3}$ on the grid points of the final mesh was stored in order to allow its interpolation at any value of time along the characteristics. The RADAU5 integrator could compute $G_{4}$ and $G_{5}$ as a succession of initial value problems (and afterwards higher-order derivatives, see below), preventing numerical "blow-up".

Results for systems I, II, and III are presented in Figure 5.

Number-average and weight-average molecular weights of sol are obtained through:

$\bar{M}_{\mathrm{n}}=\frac{M_{M_{1}} G_{4}(\mathbf{1})+M_{M_{2}} G_{5}(\mathbf{1})}{G(\mathbf{1})}$

$\bar{M}_{\mathrm{w}}=\frac{M_{M_{1}}^{2} G_{44}(\mathbf{1})+2 M_{M_{1}} M_{M_{2}} G_{45}(\mathbf{1})+M_{M_{2}}^{2} G_{55}(\mathbf{1})}{M_{M_{1}} G_{4}(\mathbf{1})+M_{M_{2}} G_{5}(\mathbf{1})}$

Molecular masses of monomers were chosen as $M_{M_{1}}=$ 104 and $M_{M_{2}}=130$ (same as styrene and divinylbenzene).

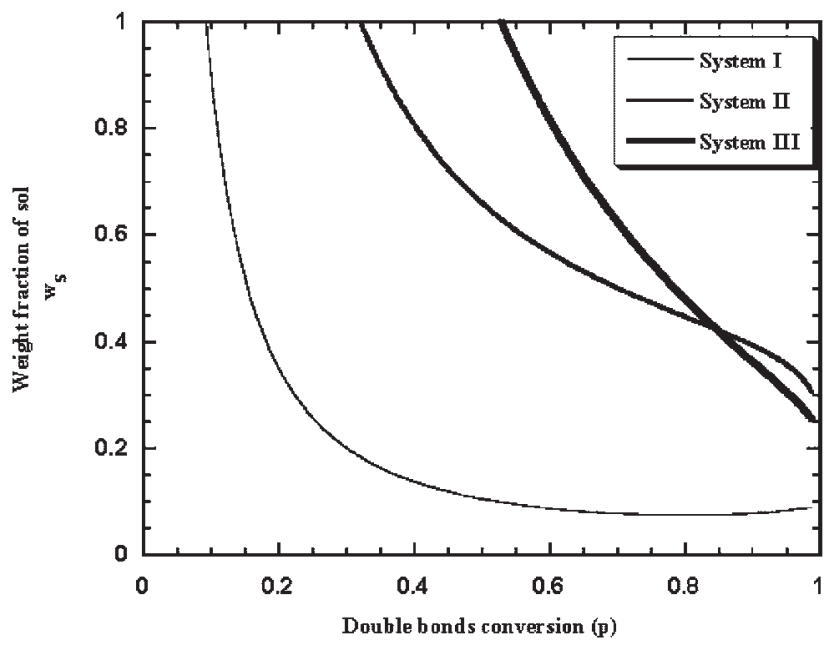

Figure 5. Fraction of sol vs. conversion of double bonds in batch polymerization of model systems I-III.
Average overall degrees of polymerization $\bar{x}_{\mathrm{n}}$ and $\bar{x}_{\mathrm{w}}$ can be obtained through Equation (47) and (48) setting molecular weights of monomers equal to 1 instead of their true values.

The system of ordinary differential equations to be integrated along characteristics in order to compute the second derivatives of $G$ (leading to the second-order moments of chain length distribution) is presented in Table 2.

Notice there is no need to use "closure assumptions" as in most proposed approximate methods. This is a consequence of not using the pseudo steady state hypothesis for radical concentrations.

Before gelation, the trivial branch $\mathbf{s}=\mathbf{1}$ is the correct solution of the two-point boundary value problem for characteristics, so it is only necessary to set the Laplace parameters $s_{j}$ equal to a constant value of 1 .

Numerical results for systems I-III are presented in Figure 6 and 7.

\section{Comparison with Predictions Using Tobita-Hamielec's Pseudo-Kinetic Rate Method}

A few approximate methods of prediction of average molecular weights and sol fraction in non-linear free radical polymerization have been proposed over the past few years. ${ }^{[14-17]}$ It is important to check those predictions with our new numerical method.

We have thus computed number-average and weightaverage molecular degrees of polymerization for systems I-III both before and after gelation using Tobita-Hamielec's pseudo-kinetic rate method ${ }^{[14,15]}$ (by far the fastest and easiest to implement) and the corresponding results are presented in Figure 8 and 9.

Agreement is fairly good for the "ideal" system I, except for high conversion.

However, for systems II and III, predicted gel points are rather different, and the curves for molecular weights and sol fraction diverge considerably from the more exact mathematical predictions developed in this paper. Moreover, we have found a slight inconsistency between pseudokinetic method predictions before and after gel point, which is especially conspicuous with system II, as can be observed in Figure 8 and 9b.

\section{Discussion}

The numerical technique presented here could yield a mathematical solution to modeling of simple non-linear free radical polymerizations with no need to use many simplifications with non-universal applicability, such as neglecting multifunctional radicals and using the pseudosteady state hypothesis. A less evident approximation is neglecting the fact that fragments of the same molecule come from gluing other molecules formed at possibly very 
Table 2. Equations of change of second order derivatives of $G$ along characteristics.

$$
\begin{aligned}
\frac{\mathrm{d} G_{11}}{\mathrm{~d} t}= & k_{\mathrm{I}_{1}} s_{1} R_{0} M_{1}+k_{\mathrm{p}_{12}} \frac{s_{2} s_{3}}{s_{1}} M_{2}\left(G_{1}-2 G_{11}\right)+k_{\mathrm{p}_{21}} \frac{s_{1}}{s_{2}} M_{1}\left(G_{2}+2 G_{12}\right) \\
& +k_{\mathrm{p}_{13}} \frac{s_{2}}{s_{1} s_{3}}\left(G_{1} G_{3}-2 G_{11} G_{3}-2 G_{13} G_{1}+2 G_{11} G_{13}\right)+2 \frac{k_{\mathrm{p}_{23}}}{s_{3}} G_{12} G_{13} \\
& +\frac{k_{\mathrm{tc}_{11}}}{s_{1}^{2}}\left(4 G_{1}^{2}-8 G_{11} G_{1}+2 G_{11}^{2}\right)+\frac{k_{\mathrm{tc}_{12}}}{s_{1} s_{2}}\left(G_{1} G_{2}-2 G_{11} G_{2}-2 G_{12} G_{1}+2 G_{11} G_{12}\right) \\
& +\frac{2 k_{\mathrm{tc}_{22}}}{s_{2}^{2}} G_{12}^{2}+2 \frac{k_{\mathrm{td}_{11}}}{s_{1}} A_{1}\left(G_{1}-2 G_{11}\right)+\frac{k_{\mathrm{td}_{12}}}{s_{1}} A_{2}\left(G_{1}-2 G_{11}\right)
\end{aligned}
$$

$$
\begin{aligned}
\frac{\mathrm{d} G_{12}}{\mathrm{~d} t}= & k_{\mathrm{I}_{3}} \frac{s_{2}}{s_{3}} R_{0} G_{13}+k_{\mathrm{p}_{12}} \frac{s_{2} s_{3}}{s_{1}} M_{2}\left(G_{11}-G_{21}-G_{1}\right)+k_{\mathrm{p}_{21}} \frac{s_{1}}{s_{2}} M_{1}\left(G_{22}-G_{12}-G_{2}\right) \\
& +k_{\mathrm{p}_{13}} \frac{s_{2}}{s_{1} s_{3}}\left(-G_{1} G_{3}-G_{21} G_{3}-G_{23} G_{1}+G_{11} G_{3}+G_{13} G_{1}+G_{11} G_{23}+G_{13} G_{21}\right) \\
& +\frac{k_{\mathrm{p}_{23}}}{s_{3}}\left(G_{12} G_{23}+G_{13} G_{22}\right)+\frac{k_{\mathrm{tc}_{11}}}{s_{1}^{2}}\left(2 G_{11} G_{21}-4 G_{21} G_{1}\right) \\
& +\frac{k_{\mathrm{tc}_{12}}}{s_{1} s_{2}}\left(G_{1} G_{2}-G_{21} G_{2}-G_{22} G_{1}-G_{11} G_{2}-G_{12} G_{1}+G_{11} G_{22}+G_{12} G_{21}\right) \\
& +\frac{k_{\mathrm{tc}_{22}}}{s_{2}^{2}}\left(2 G_{12} G_{22}-4 G_{12} G_{2}\right)-2 \frac{k_{\mathrm{td}_{11}}}{s_{1}} A_{1} G_{21}-k_{\mathrm{td}_{12}}\left(\frac{A_{2} G_{21}}{s_{1}}+\frac{A_{1} G_{12}}{s_{2}}\right)-2 \frac{k_{\mathrm{td}_{22}}}{s_{2}} A_{2} G_{12}
\end{aligned}
$$

$$
\begin{aligned}
\frac{\mathrm{d} G_{13}}{\mathrm{~d} t}= & -k_{\mathrm{I}_{3}} \frac{s_{2}}{s_{3}} R_{0} G_{13}+k_{\mathrm{p}_{12}} \frac{s_{2} s_{3}}{s_{1}} M_{2}\left(G_{11}-G_{31}-G_{1}\right)+k_{\mathrm{p}_{21}} \frac{s_{1}}{s_{2}} M_{1} G_{32}+k_{\mathrm{p}_{22}} s_{3} M_{2} G_{12} \\
& +k_{\mathrm{p}_{13}} \frac{s_{2}}{s_{1} s_{3}}\left(G_{1} G_{3}-G_{31} G_{3}-G_{33} G_{1}-G_{11} G_{3}-G_{13} G_{1}+G_{11} G_{33}+G_{13} G_{31}\right) \\
& +\frac{k_{\mathrm{p}_{23}}}{s_{3}}\left(-G_{12} G_{3}-G_{13} G_{2}+G_{12} G_{33}+G_{13} G_{32}\right) \\
& +\frac{k_{\mathrm{tc}_{11}}}{s_{1}^{2}}\left(2 G_{11} G_{31}-4 G_{31} G_{1}\right)+\frac{k_{\mathrm{tc}_{12}}}{s_{1} s_{2}}\left(-G_{31} G_{2}-G_{32} G_{1}+G_{11} G_{32}+G_{12} G_{31}\right) \\
& +2 \frac{k_{\mathrm{tc}_{22}}}{s_{2}^{2}} G_{12} G_{32}-2 \frac{k_{\mathrm{td}_{11}}}{s_{1}} A_{1} G_{31}-\frac{k_{\mathrm{td}_{12}}}{s_{1}} A_{2} G_{31}
\end{aligned}
$$

$$
\begin{aligned}
\frac{\mathrm{d} G_{14}}{\mathrm{~d} t}= & k_{\mathrm{I}_{1}} s_{1} R_{0} M_{1}+k_{\mathrm{p}_{11}} M_{1} G_{11}-k_{\mathrm{p}_{12}} \frac{s_{2} s_{3}}{s_{1}} M_{2} G_{41}+k_{\mathrm{p}_{21}} \frac{s_{1}}{s_{2}} M_{1}\left(G_{42}+G_{2}+G_{12}\right) \\
& +k_{\mathrm{p}_{13}} \frac{s_{2}}{s_{1} s_{3}}\left(G_{11} G_{43}+G_{13} G_{41}-G_{41} G_{3}-G_{43} G_{1}\right)+\frac{k_{\mathrm{p}_{23}}}{s_{3}}\left(G_{12} G_{43}+G_{13} G_{42}\right) \\
& +\frac{k_{\mathrm{tc}_{11}}}{s_{1}^{2}}\left(2 G_{11} G_{41}-4 G_{14} G_{1}\right)+\frac{k_{\mathrm{tc}_{12}}}{s_{1} s_{2}}\left(-G_{41} G_{2}-G_{42} G_{1}+G_{11} G_{42}+G_{12} G_{41}\right) \\
& +2 \frac{k_{\mathrm{tc}_{22}}}{s_{2}^{2}} G_{12} G_{42}-2 \frac{k_{\mathrm{td}_{11}}}{s_{1}} A_{1} G_{41}-\frac{k_{\mathrm{td}_{12}}}{s_{1}} A_{2} G_{41}
\end{aligned}
$$

$$
\begin{aligned}
\frac{\mathrm{d} G_{15}}{\mathrm{~d} t}= & k_{\mathrm{p}_{12}} \frac{s_{2} s_{3}}{s_{1}} M_{2}\left(G_{11}-G_{51}-G_{1}\right)+k_{\mathrm{p}_{21}} \frac{s_{1}}{s_{2}} M_{1} G_{52}+k_{\mathrm{p}_{22}} s_{3} M_{2} G_{12} \\
& +k_{\mathrm{p}_{13}} \frac{s_{2}}{s_{1} s_{3}}\left(G_{11} G_{53}+G_{13} G_{51}-G_{51} G_{3}-G_{53} G_{1}\right)+\frac{k_{\mathrm{p}_{23}}}{s_{3}}\left(G_{12} G_{53}+G_{13} G_{52}\right) \\
& +\frac{k_{\mathrm{tc}_{11}}}{s_{1}^{2}}\left(2 G_{11} G_{51}-4 G_{51} G_{1}\right)+\frac{k_{\mathrm{tc}_{12}}}{s_{1} s_{2}}\left(-G_{51} G_{2}-G_{52} G_{1}+G_{11} G_{52}+G_{12} G_{51}\right) \\
& +2 \frac{k_{\mathrm{tc}_{22}}}{s_{2}^{2}} G_{12} G_{52}-2 \frac{k_{\mathrm{td}_{11}}}{s_{1}} A_{1} G_{51}-\frac{k_{\mathrm{td}_{12}}}{s_{1}} A_{2} G_{51}
\end{aligned}
$$


Table 2. (Continued)

$$
\begin{aligned}
\frac{\mathrm{d} G_{22}}{\mathrm{~d} t}= & k_{\mathrm{I}_{2}} s_{2} s_{3} R_{0} M_{2}+k_{\mathrm{I}_{3}} \frac{s_{2}}{s_{3}} R_{0}\left(2 G_{23}+G_{3}\right)+k_{\mathrm{p}_{12}} \frac{s_{2} s_{3}}{s_{1}} M_{2}\left(2 G_{21}+G_{1}\right) \\
& +k_{\mathrm{p}_{21}} \frac{s_{1}}{s_{2}} M_{1}\left(G_{2}-2 G_{22}\right)+k_{\mathrm{p}_{13}} \frac{s_{2}}{s_{1} s_{3}}\left(G_{1} G_{3}+2 G_{21} G_{3}+2 G_{23} G_{1}+2 G_{21} G_{23}\right)+2 \frac{k_{\mathrm{p}_{23}}}{s_{3}} G_{22} G_{23} \\
& +\frac{2 k_{\mathrm{tc}_{11}}}{s_{1}^{2}} G_{21}^{2}+\frac{k_{\mathrm{tc}_{12}}}{s_{1} s_{2}}\left(G_{1} G_{2}-2 G_{21} G_{2}-2 G_{22} G_{1}+2 G_{21} G_{22}\right) \\
& +\frac{k_{\mathrm{tc}_{22}}}{s_{2}^{2}}\left(4 G_{2}^{2}-8 G_{22} G_{2}+2 G_{22}^{2}\right)+2 \frac{k_{\mathrm{td}_{12}}}{s_{2}} A_{1}\left(G_{2}-2 G_{22}\right)+\frac{2 k_{\mathrm{td}_{22}}}{s_{2}} A_{2}\left(G_{2}-2 G_{22}\right) \\
\frac{\mathrm{d} G_{23}}{\mathrm{~d} t} & k_{\mathrm{I}_{2}} s_{2} s_{3} R_{0} M_{2}+k_{\mathrm{I}_{3}} \frac{s_{2}}{s_{3}} R_{0}\left(G_{33}-G_{23}-G_{3}\right)+k_{\mathrm{p}_{12}} \frac{s_{2} s_{3}}{s_{1}} M_{2}\left(G_{31}+G_{21}+G_{1}\right)-k_{\mathrm{p}_{21}} \frac{s_{1}}{s_{2}} M_{1} G_{32} \\
& +k_{\mathrm{p}_{22}} s_{3} M_{2} G_{22}+k_{\mathrm{p}_{13}} \frac{s_{2}}{s_{1} s_{3}}\left(-G_{1} G_{3}+G_{31} G_{3}+G_{33} G_{1}-G_{21} G_{3}-G_{23} G_{1}+G_{21} G_{33}+G_{23} G_{31}\right) \\
& +\frac{k_{\mathrm{p}_{23}}}{s_{3}}\left(-G_{22} G_{3}-G_{23} G_{2}+G_{22} G_{33}+G_{23} G_{32}\right)+\frac{2 k_{\mathrm{tc}_{11}}}{s_{1}^{2}} G_{21} G_{31}+\frac{k_{\mathrm{tc}_{22}}}{s_{2}^{2}} G_{32}\left(2 G_{22}-4 G_{2}\right) \\
& +\frac{k_{\mathrm{tc}_{12}}}{s_{1} s_{2}}\left(-G_{31} G_{2}-G_{32} G_{1}+G_{21} G_{32}+G_{22} G_{31}\right)-\frac{k_{\mathrm{td}_{12}}}{s_{2}} A_{1} G_{32}-\frac{2 k_{\mathrm{td}_{22}} A_{2} G_{32}}{s_{2}}
\end{aligned}
$$

$$
\begin{aligned}
\frac{\mathrm{d} G_{24}}{\mathrm{~d} t}= & k_{\mathrm{I}_{3}} \frac{s_{2}}{s_{3}} R_{0} G_{43}+k_{\mathrm{p}_{11}} M_{1} G_{21}+k_{\mathrm{p}_{12}} \frac{s_{2} s_{3}}{s_{1}} M_{2} G_{41}+k_{\mathrm{p}_{21}} \frac{s_{1}}{s_{2}} M_{1}\left(G_{22}-G_{42}-G_{2}\right) \\
& +k_{\mathrm{p}_{13}} \frac{s_{2}}{s_{1} s_{3}}\left(G_{41} G_{3}+G_{43} G_{1}+G_{21} G_{43}+G_{23} G_{41}\right)+\frac{k_{\mathrm{p}_{23}}}{s_{3}}\left(G_{22} G_{43}+G_{23} G_{42}\right) \\
& +\frac{2 k_{\mathrm{tc}_{11}}}{s_{1}^{2}} G_{21} G_{41}+\frac{k_{\mathrm{tc}_{12}}}{s_{1} s_{2}}\left(-G_{41} G_{2}-G_{42} G_{1}+G_{21} G_{42}+G_{22} G_{41}\right)+\frac{k_{\mathrm{tc}_{22}}}{s_{2}^{2}} G_{42}\left(2 G_{22}-4 G_{2}\right) \\
& -\frac{k_{\mathrm{td}_{12}}}{s_{2}} A_{1} G_{42}-\frac{2 k_{\mathrm{td}_{22}}}{s_{2}} A_{2} G_{42}
\end{aligned}
$$

$$
\begin{aligned}
\frac{\mathrm{d} G_{25}}{\mathrm{~d} t}= & k_{\mathrm{I}_{2}} s_{2} s_{3} R_{0} M_{2}+k_{\mathrm{I}_{3}} \frac{s_{2}}{s_{3}} R_{0} G_{53}+k_{\mathrm{p}_{12}} \frac{s_{2} s_{3}}{s_{1}} M_{2}\left(G_{51}+G_{21}+G_{1}\right)-k_{\mathrm{p}_{21}} \frac{s_{1}}{s_{2}} M_{1} G_{52} \\
& +k_{\mathrm{p}_{22}} s_{3} M_{2} G_{22}+k_{\mathrm{p}_{13}} \frac{s_{2}}{s_{1} s_{3}}\left(G_{51} G_{3}+G_{53} G_{1}+G_{21} G_{53}+G_{23} G_{51}\right)+\frac{k_{\mathrm{p}_{23}}}{s_{3}}\left(G_{22} G_{53}+G_{23} G_{52}\right) \\
& +\frac{2 k_{\mathrm{tc}_{11}}}{s_{1}^{2}} G_{21} G_{51}+\frac{k_{\mathrm{tc}_{12}}}{s_{1} s_{2}}\left(-G_{51} G_{2}-G_{52} G_{1}+G_{21} G_{52}+G_{22} G_{51}\right)+\frac{k_{\mathrm{tc}_{22}}}{s_{2}^{2}} G_{52}\left(2 G_{22}-4 G_{2}\right) \\
& -\frac{k_{\mathrm{td}_{12}}}{s_{2}} A_{1} G_{52}-\frac{2 k_{\mathrm{td}_{22}}}{s_{2}} A_{2} G_{52}
\end{aligned}
$$

$$
\begin{aligned}
\frac{\mathrm{d} G_{33}}{\mathrm{~d} t}= & k_{\mathrm{I}_{2}} s_{2} s_{3} R_{0} M_{2}+k_{\mathrm{I}_{3}} \frac{s_{2}}{s_{3}} R_{0}\left(G_{3}-2 G_{33}\right)+k_{\mathrm{p}_{12}} \frac{s_{2} s_{3}}{s_{1}} M_{2}\left(2 G_{31}+G_{1}\right) \\
& +k_{\mathrm{p}_{22}} s_{3} M_{2}\left(2 G_{32}+G_{2}\right)+k_{\mathrm{p}_{13}} \frac{s_{2}}{s_{1} s_{3}}\left(G_{1} G_{3}-2 G_{31} G_{3}-2 G_{33} G_{1}+2 G_{31} G_{33}\right) \\
& +\frac{k_{\mathrm{p}_{23}}}{s_{3}}\left(G_{2} G_{3}-2 G_{32} G_{3}-2 G_{33} G_{2}+2 G_{32} G_{33}\right)+\frac{2 k_{\mathrm{tc}_{11}}}{s_{1}^{2}} G_{31}^{2}+\frac{2 k_{\mathrm{tc}_{12}}}{s_{1} s_{2}} G_{31} G_{32}+\frac{2 k_{\mathrm{tc}_{22}}}{s_{2}^{2}} G_{32}^{2}
\end{aligned}
$$

$$
\begin{aligned}
\frac{\mathrm{d} G_{34}}{\mathrm{~d} t}= & -k_{\mathrm{I}_{3}} \frac{s_{2}}{s_{3}} R_{0} G_{43}+k_{\mathrm{p}_{11}} M_{1} G_{31}+k_{\mathrm{p}_{12}} \frac{s_{2} s_{3}}{s_{1}} M_{2} G_{41}+k_{\mathrm{p}_{21}} \frac{s_{1}}{s_{2}} M_{1} G_{32} \\
& +k_{\mathrm{p}_{22}} s_{3} M_{2} G_{42}+k_{\mathrm{p}_{13}} \frac{s_{2}}{s_{1} s_{3}}\left(G_{31} G_{43}+G_{33} G_{41}-G_{41} G_{3}-G_{43} G_{1}\right) \\
& +\frac{k_{\mathrm{p}_{23}}}{s_{3}}\left(G_{32} G_{43}+G_{33} G_{42}-G_{42} G_{3}-G_{43} G_{2}\right)+\frac{2 k_{\mathrm{tc}_{11}}}{s_{1}^{2}} G_{31} G_{41}+\frac{k_{\mathrm{tc}_{12}}}{s_{1} s_{2}}\left(G_{31} G_{42}+G_{32} G_{41}\right)+\frac{2 k_{\mathrm{tc}_{22}}}{s_{2}^{2}} G_{32} G_{42}
\end{aligned}
$$




$$
\begin{aligned}
& \frac{\mathrm{d} G_{35}}{\mathrm{~d} t}=k_{\mathrm{I}_{2}} s_{2} s_{3} R_{0} M_{2}-k_{\mathrm{I}_{3}} \frac{s_{2}}{s_{3}} R_{0} G_{53}+k_{\mathrm{p}_{12}} \frac{s_{2} s_{3}}{s_{1}} M_{2}\left(G_{51}+G_{31}+G_{1}\right) \\
& +k_{\mathrm{p}_{22}} s_{3} M_{2}\left(G_{52}+G_{32}+G_{2}\right)+k_{\mathrm{p}_{13}} \frac{s_{2}}{s_{1} s_{3}}\left(G_{31} G_{53}+G_{33} G_{51}-G_{51} G_{3}-G_{53} G_{1}\right) \\
& +\frac{k_{\mathrm{p}_{23}}}{s_{3}}\left(G_{32} G_{53}+G_{33} G_{52}-G_{52} G_{3}-G_{53} G_{2}\right)+\frac{2 k_{\mathrm{tc}_{11}}}{s_{1}^{2}} G_{31} G_{51} \\
& +\frac{k_{\mathrm{tc}_{12}}}{s_{1} s_{2}}\left(G_{31} G_{52}+G_{32} G_{51}\right)+\frac{2 k_{\mathrm{tc}_{22}}}{s_{2}^{2}} G_{32} G_{52} \\
& \frac{\mathrm{d} G_{44}}{\mathrm{~d} t}=k_{\mathrm{I}_{1}} s_{1} R_{0} M_{1}+k_{\mathrm{p}_{11}} M_{1}\left(2 G_{41}+G_{1}\right)+k_{\mathrm{p}_{21}} \frac{s_{1}}{s_{2}} M_{1}\left(2 G_{42}+G_{2}\right) \\
& +2 k_{\mathrm{p}_{13}} \frac{s_{2}}{s_{1} s_{3}} G_{41} G_{43}+\frac{2 k_{\mathrm{p}_{23}}}{s_{3}} G_{42} G_{43}+\frac{2 k_{\mathrm{tc}_{11}}}{s_{1}^{2}} G_{41}^{2}+\frac{2 k_{\mathrm{tc}_{12}}}{s_{1} s_{2}} G_{41} G_{42}+\frac{2 k_{\mathrm{tc}_{22}}}{s_{2}^{2}} G_{42}^{2} \\
& \frac{\mathrm{d} G_{45}}{\mathrm{~d} t}=k_{\mathrm{p}_{11}} M_{1} G_{51}+k_{\mathrm{p}_{12}} \frac{s_{2} s_{3}}{s_{1}} M_{2} G_{41}+k_{\mathrm{p}_{21}} \frac{s_{1}}{s_{2}} M_{1} G_{52}+k_{\mathrm{p}_{22}} s_{3} M_{2} G_{42} \\
& +k_{\mathrm{p}_{13}} \frac{s_{2}}{s_{1} s_{3}}\left(G_{41} G_{53}+G_{43} G_{51}\right)+\frac{k_{\mathrm{p}_{23}}}{s_{3}}\left(G_{42} G_{53}+G_{43} G_{52}\right) \\
& +\frac{2 k_{\mathrm{tc}_{11}}}{s_{1}^{2}} G_{41} G_{51}+\frac{k_{\mathrm{tc}_{12}}}{s_{1} s_{2}}\left(G_{41} G_{52}+G_{42} G_{51}\right)+\frac{2 k_{\mathrm{tc}_{22}}}{s_{2}^{2}} G_{42} G_{52} \\
& \frac{\mathrm{d} G_{55}}{\mathrm{~d} t}=k_{\mathrm{I}_{2}} s_{2} s_{3} R_{0} M_{2}+k_{\mathrm{p}_{12}} \frac{s_{2} s_{3}}{s_{1}} M_{2}\left(2 G_{51}+G_{1}\right)+k_{\mathrm{p}_{22}} s_{3} M_{2}\left(2 G_{52}+G_{2}\right) \\
& +2 k_{\mathrm{p}_{13}} \frac{s_{2}}{s_{1} s_{3}} G_{51} G_{53}+\frac{2 k_{\mathrm{p}_{23}}}{s_{3}} G_{52} G_{53}+\frac{2 k_{\mathrm{tc}_{11}}}{s_{1}^{2}} G_{51}^{2}+\frac{2 k_{\mathrm{tc}_{12}}}{s_{1} s_{2}} G_{51} G_{52}+\frac{2 k_{\mathrm{tc}_{22}}}{s_{2}^{2}} G_{52}^{2}
\end{aligned}
$$

different times. There is no uniformity of link probabilities inside the same molecule (as would happen in equilibrium reversible polymerization) and description by a simple Markovian process, which could be treated by the theory of branching processes, is not exact. This is one of the main

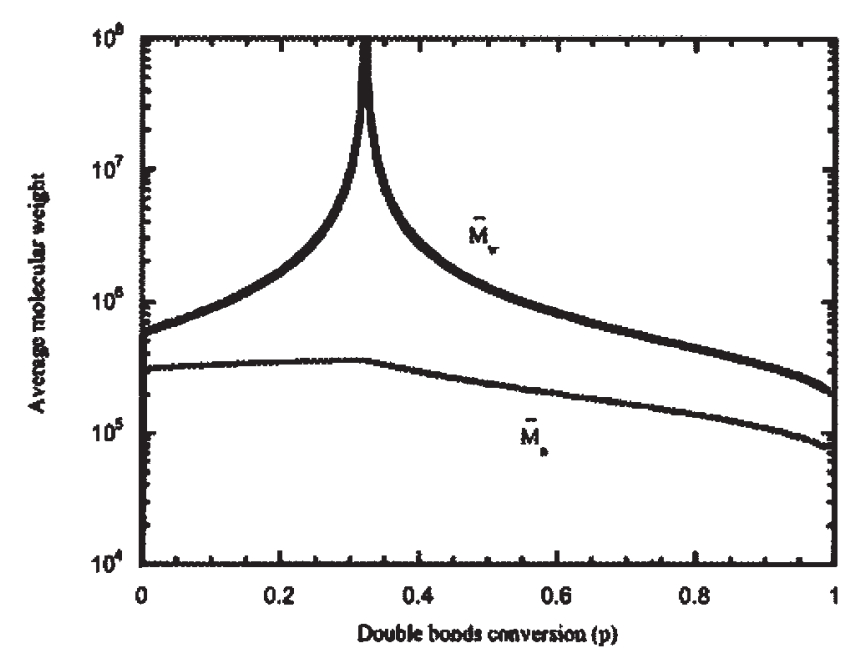

Figure 6. Number-average and weight-average molecular weights of sol in batch non-linear free radical polymerizations of model system II. alerts already formulated in ref. ${ }^{[1]}$ (only when gelation conversion is low should a good agreement with usual approximations be expected). It is no wonder that the pseudo-kinetic rate method fails to give an acceptable quantitative approximation of the behavior of molecular weight

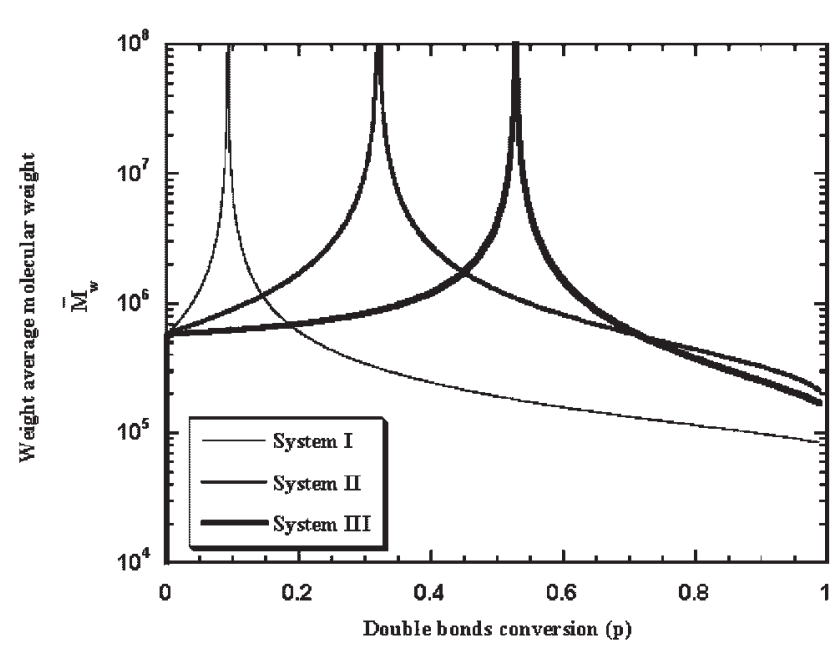

Figure 7. Weight-average molecular weights of sol in batch nonlinear free radical polymerizations of model systems I-III. 


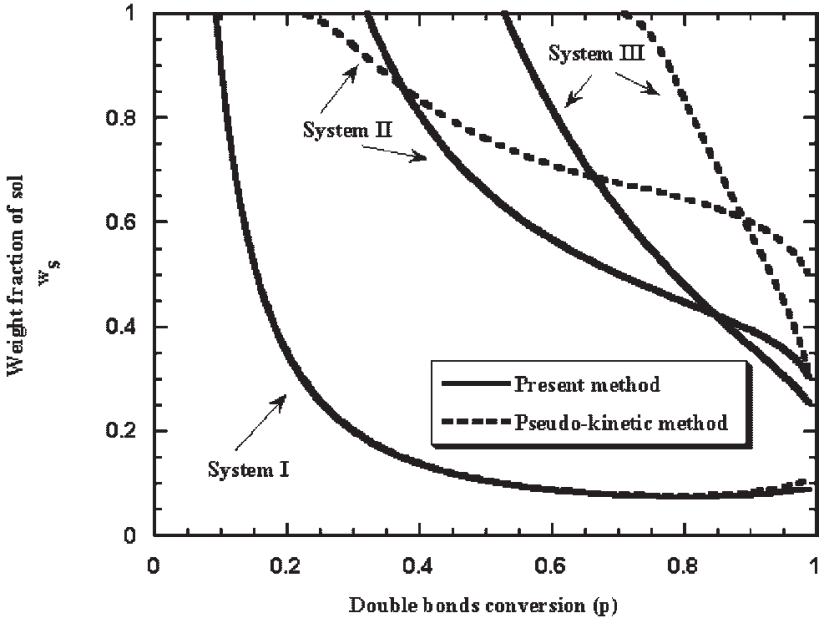

Figure 8. Comparison between predicted sol fraction in batch non-linear free radical polymerizations of model systems I-III according to Tobita-Hamielec's pseudo-kinetic method and our present approach.

or sol fraction changes along time in some circumstances, when reactivity ratios are very different from 1 .

Other researchers have described these and other polymerization systems staying in the domain of real chain lengths and aiming at obtaining if not the full multidimensional CLD, at least some of its marginal distributions, the prediction of average molecular weights becoming a "byproduct" of those computations.

Although, in theory, any distribution can be reconstructed from the knowledge of the infinite set of its moments, and this work has provided a way of computing them with comparatively little difficulty (before gelation), we do not recommend this procedure. Such Gram-Charlier series often converge slowly, and there is no way to guarantee the accuracy of the computations. A similar problem, the prediction of the shape of chromatographic peaks in linear chromatography, presents striking similarities. Villermaux ${ }^{[18]}$ proved a long time ago that numerical inversion of its Laplace transform is a fast and reliable way of solving the problem, in contrast to the series approximation. More recently, Tobita and Ito ${ }^{[19]}$ have also cast serious doubts concerning the usefulness of those series to represent the CLD of polymers.

There is no need ${ }^{[20]}$ to assume that chain length is a continuous variable, as suitable base functions of orthogonal polynomials of discrete variables can be found. But this approach faces serious problems when dealing with multidimensional distributions such as the ones discussed in these case studies: there are five kinds of groups which have to be counted for each set of isomeric polymer molecules, disregarding the way the units are connected. Indeed, commercial code PREDICI, using this principle, can only compute one-dimensional distributions. Nevertheless, with some adaptations ${ }^{[21]}$ (remembering Teymour's "numerical
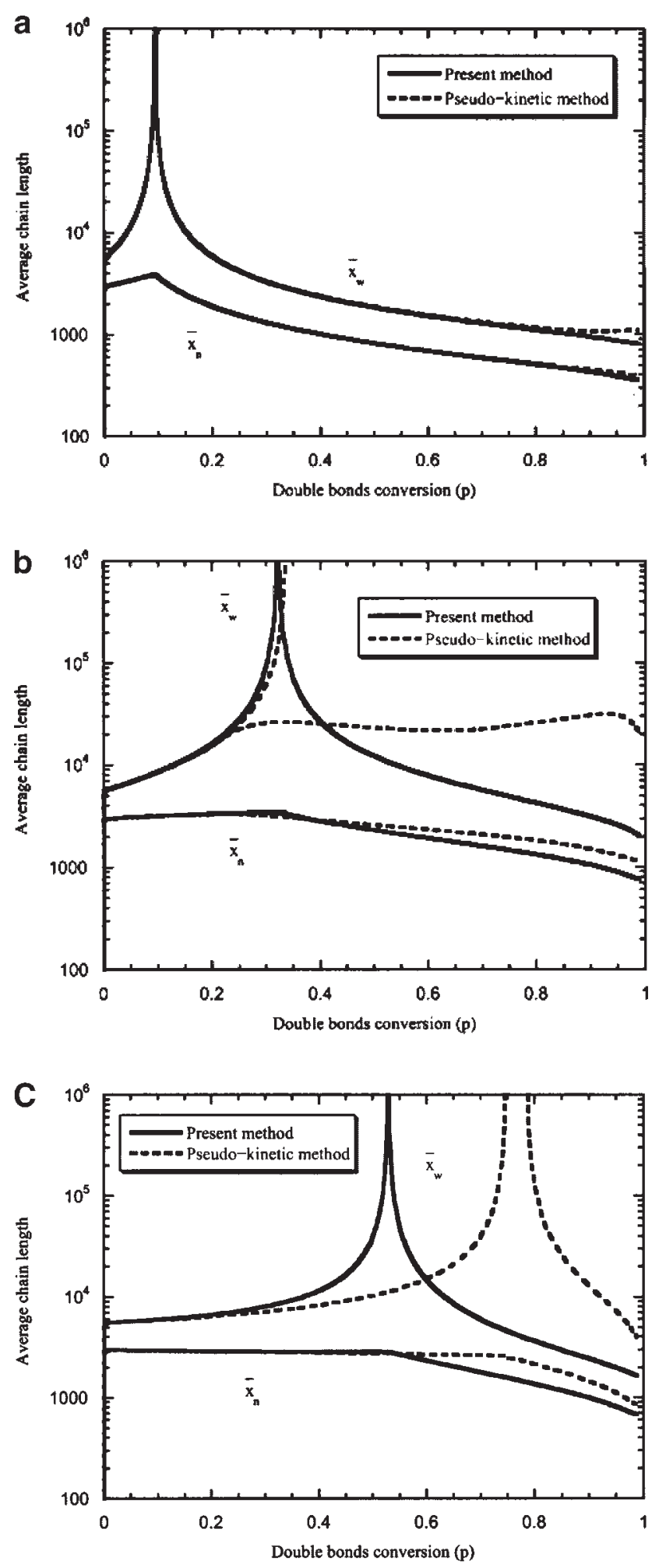

Figure 9. Comparison between predicted number-average and weight-average degrees of polymerization of sol in batch nonlinear free radical polymerizations of model systems I-III according to Tobita-Hamielec pseudo-kinetic method and present approach. (a) System I, (b) System II, (c) System III. 
fractionating" approach ${ }^{[16,22]}$ ) it could be used for modeling ethylene free radical polymerization.

Free radical polymerizations with very low concentrations of polyradicals (species with $a_{1}+a_{2}>1$ in our notation) might be amenable to a fairly accurate description with the help of the "numerical fractionating" concept. We doubt this could be valid for the kind of chemical systems discussed in this current work, but it remains an open question whether our suspicion is real.

Another possible solution for predicting CLD is the use of Monte Carlo simulation, widely used in the last decade by Tobita and Zhu. ${ }^{[23,24]}$ This approach presents some additional important advantages, such as the possibility of prediction of the distribution of molecular sizes (very difficult otherwise) or the analysis of reversible polymerization. Its main drawback lies in the computational resources needed for a reasonable accuracy. They are moderate for simple problems such as non-linear polycondensation modeling, but dealing with free-radical polymerization has been done using some approximations introduced in order to save computer time. The main difficulty lies in the very small time scales of the molecular weight growth by propagation, as compared to the time span needed to convert an appreciable amount of monomer. So, the objection raised before also applies: it is nearly as inaccurate as the theory of branching processes (no wonder that both give similar results), and a new Monte Carlo method taking care of that problem (unfortunately extremely slow) is yet to be attempted.

A comprehensive comparison with the proposed computational method would certainly be worthwhile, but it depends on the ability to drastically reduce its computing time requirements.

Nevertheless, prediction of CLD by Monte Carlo method or finite elements with the sole purpose of computing average molecular weights seems a waste of computer resources. It is unlikely that this can be done as efficiently for the same accuracy as with the present method.

Realistic models for non-linear free radical polymerization, introducing, for instance, intramolecular transfers and terminations giving rise to short sized rings, have to take into account a huge number of chemical groups. Thus, the number of variables of the system of characteristics will grow a lot and it may become quite demanding in computational resources (memory and computing speed). It can not be stated for the moment whether these problems will become easily tractable or not by the present approach, but we consider it to be worth the effort of pursuing this research in view of the first encouraging results just presented.

\section{Conclusions}

It has been possible for the first time to find a numerical solution for the equations describing the evolution of generating function of chain length distribution for nonlinear free-radical polymerization, assuming no chain length dependence of termination constant and no intramolecular reaction in finite molecules. Pseudo-steady hypothesis and other widespread simplifications are not required.

Sol fraction and number- and weight-average molecular weights were computed for monovinyl/divinyl polymerizations with different reactivity ratios.

A computer with a single Intel PIV $1.5 \mathrm{GHz}$ with $512 \mathrm{Mb}$ using public domain software was enough for solving these rather simple problems, but memory use was $60 \%$ of the available, and a more powerful machine seems needed for most systems of practical interest.

A comparison with Tobita-Hamielec's pseudo-kinetic method showed minor differences for the ideal equal reactivity system, but discrepancies are important when reactivity ratios become very different from 1 .

Acknowledgement: We acknowledge financial support by Fundação da Ciência e Tecnologia through programs Praxis (proposal 3/3.1/CEG/2525/95) and POCTI.

[1] S. I. Kuchanov, L. M. Pis'men, Vysokomol. Soyed. 1971, A13, 2035

[2] M. R. P. F. N. Costa, R. C. S. Dias, Chem. Eng. Sci. 1994, 49, 491.

[3] M. R. P. F. N. Costa, R. C. S. Dias, "New Developments in Kinetical Modeling of Complex Non-linear Polymerizations", in: 5th International Workshop on Polymer Reaction Engineering, K. H. Reichert, H.-U. Moritz, Eds., VCH, Weinheim 1995, pp. 523-530.

[4] R. Courant, D. Hilbert, "Methods of Mathematical Physics, Vol. II-Partial Differential Equations", 1st edition, WileyInterscience, New York 1962, pp. 97-105.

[5] P. Deuflhard, F. Bornemann, "Scientific Computing with Ordinary Differential Equations", 1st edition, Springer, New York 2002, p. 390.

[6] E. Hairer, G. Wanner, "Solving Ordinary Differential Equations II', 2nd revised edition, Springer, New York 2002, p. 1.

[7] U. Ascher, L. R. Petzold, "Computer Methods for Ordinary Differential and Differential-Algebraic Equations", 1st edition, SIAM 1998, pp. 50-51.

[8] E. Hairer, G. Wanner, "Solving Ordinary Differential Equations II', 2nd revised edition, Springer, New York 2002, pp. 566-574.

[9] H. B. Keller, "Numerical Methods for Two-Point BoundaryValue Problems", 2nd edition, Dover, Mineola, New York 1992, pp. 61.

[10] J. R. Cash, G. Moore, R. W. Wright, J. Comp. Phys. 1995, 122, 266.

[11] Z. Bashir-Ali, J. R. Cash, H. H. M. Silva, Comput. Math. Appl. 1998, 36, 59. 
[12] J. R. Cash, G. Moore, R. W. Wright, ACM Trans. Math. Soft. 2001, 27, 245.

[13] G. Moad, D. H. Solomon, "The Chemistry of Free Radical Polymerization", 1st edition, Elsevier, New York 1995, pp. 191 and 209.

[14] H. Tobita, A. E. Hamielec, Macromolecules 1989, 23, 3098.

[15] H. Tobita, A. E. Hamielec, "Crosslinking Kinetics in FreeRadical Copolymerization", in: 3rd International Workshop on Polymer Reaction Engineering, K. H. Reichert, W. Geiseler, Eds., VCH, Weinheim 1989, pp. 43-83.
[16] F. Teymour, J. D. Campbell, Macromolecules 1994, 27, 2460.

[17] H. Tobita, Macromol. Theory Simul. 1998, 7, 675.

[18] J. Villermaux, J. Chromatogr. 1973, 83, 205.

[19] H. Tobita, K. Ito, Polym. React. Eng. 1993, 1, 407.

[20] U. Budde, M. Wulkow, Chem. Eng. Sci. 1991, 46, 497.

[21] P. D. Iedema, M. Wulkow, H. C. J. Hoefsloot, Macromolecules 2000, 33, 7173.

[22] P. Pladis, C. Kiparissides, Chem. Eng. Sci. 1998, 53, 3315.

[23] H. Tobita, J. Polym. Sci., Part B: Polym. Phys. 1993, 31, 1363.

[24] H. Tobita, S. Zhu, J. Polym. Sci., Part B: Polym. Phys. 1996, 34, 2099. 